\title{
Multi Domain Design of Fluid Machinery
}

\author{
Andrea Perrone ${ }^{1}$, Luigi De Napoli ${ }^{1}$, Clara Nino $^{2}$ and Antonino Morabito ${ }^{2}$ \\ 1. DIMEG (Department of Mechanical, Energy and Management Engineering), Università della Calabria, Rende 87036, Italy \\ 2. Research New Technologies-DNMP srls, Rende 87036, Italy
}

\begin{abstract}
The scope of the paper is the design of a fluid machinery with a multiphysics procedure, through CAD (computer-aided design)-CAE (computer-aided engineering)-CAx (computer-aided technologies) tools. The study of a wind turbine is a perfect example of a project that requires an interdisciplinary team design, in fact for such a project mechanical structure, and CFD (computational fluid dynamics) designers are necessary. In addition, in the last years, the multi domain design has been supported by the continuous development of software for computer-aided design and the unceasing increase of the connection between performances and computer costs: the final aim is to propose the instruments that assist the designer throughout all the steps of the project. The multi domain design, illustrated in this article, is based on how to design the wind turbine, and it is not about process management and manufacturability. In particular, in the article, the emphasis will be placed on the integration between CAD and mathematical software of general type and on the CAD-CAE integration.
\end{abstract}

Key words: Multi domain design, fluid machinery, CAE, CAD, ALE (arbitrary Lagrangian-Eulerian).

\section{Introduction}

In the study of fluid machinery, difficulties consist in having kinetic-dynamic analysis, modal analysis and computational fluid dynamic issues at the same time, so it is necessary to use integrated instruments to keep under control everything contemporaneously [1], in order to achieve the proper embodiment design.

In Fig. 1, an example of conceptual map for a fluid machinery is illustrated.

In order to design a fluid machinery like a wind turbine with multi domain design in a virtual design environment, it will be necessary to make a multiphysics model able to solve many issues.

The preliminary sizing of the turbine is performed on the base of this model, then, in order to set up the embodiment design, the integration of mathematical and CAD (computer-aided design) model is accomplished, so that the project dimensions are bi-directionally linked between CAD and software/mathematical model (Fig. 2).

Corresponding author: Luigi De Napoli, assistant professor, Ph.D., research fields: CAD, CAE and sustainable product development. E-mail: luigi.denapoli@unical.it.

\section{Modelling through Integrated CAE Methodology}

In order to model a wind turbine with a CAE (computer-aided engineering) methodology, the design strategy is based on CFD (computer-aided design) combined with a moving mesh technique, FEM (finite element method) and ODE (ordinary differential equation).

So, it will be necessary to create a suitable multiphysics model, that, at the same time, is able:

- to solve Navier-Stokes equations [2];

- to calculate the stress on the structure and the flow-induced vibrations, using as input the stress produced by fluid on the turbine blade;

- to solve the equations that, using angular velocity and acceleration of the turbine and its geometrical and mass properties as entry data, permit by means of the proper mesh to describe the turbine movement (ALE mesh, i.e., arbitrary Lagrangian-Eulerian method, is adopted [3]); so the model will contain all the 


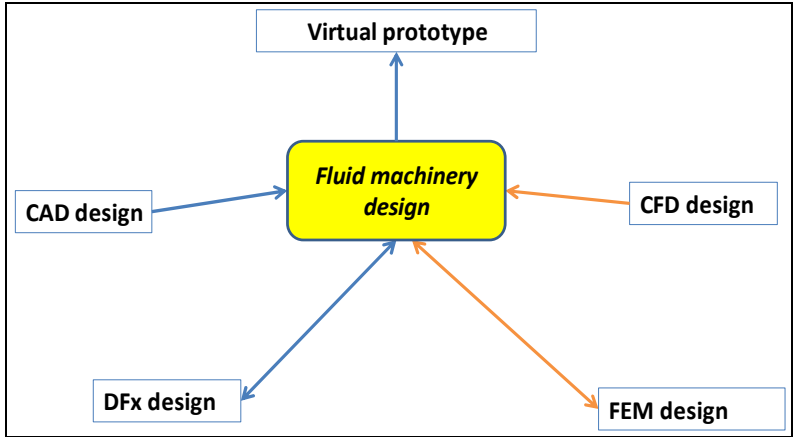

Fig. 1 Multi domain design of a wind turbine.

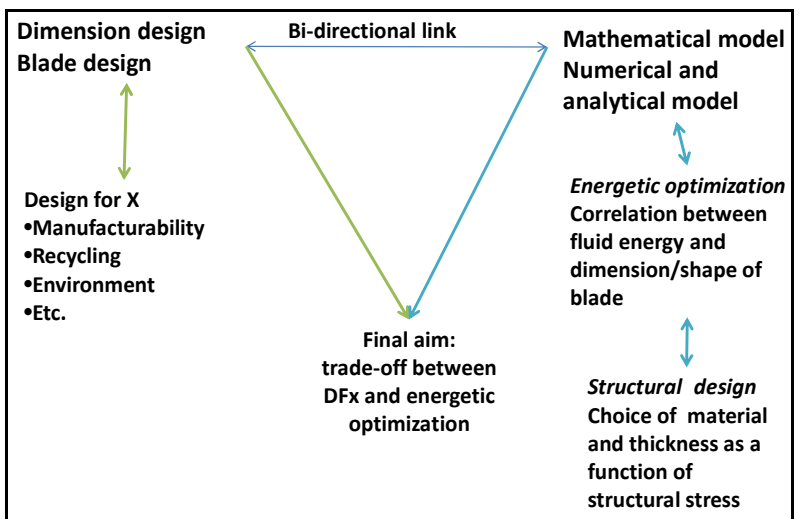

Fig. 2 Integration between CAD and mathematical software.

information of the fluid-structure interaction in every moment [4].

\subsection{Multiphysics Model of a Wind Turbine}

In order to solve the problems explained above, it will be necessary to create a multiphysics model composed by:

- computational fluid dynamic module;

- structural mechanical module;

- ODE module;

- moving mesh module [5].

These four modules are cascade connected and work cyclically—every cycle represents a time instant: the cycle end when the motion is fully developed, that is when the angular speed of the $i$ th cycle is lower than that of the $i$ th- 1 cycle.

At the beginning of the process, the CFD module receives the wind speed and the geometry of turbine blades as input, and calculates the fluid pressure on the blades as output.
The structural mechanical module has the pressure on the blades as input (that is the output of CFD module), in addition to the blade geometry and to mechanical characteristics of its material. As output, this module will give tension and deformation values on the blades and the resultant couple moment on them (Fig. 3).

Instead, through ODE module, it is possible to simulate the turbine dynamics. This module will have as input the law of motion (Eq. (1)), the polar moment of inertia and the resultant moment on the blades (that is the output of the structural module). As output, the ODE module will give the rotation angle, the angular velocity and the angular acceleration:

$$
\ddot{\vartheta}=\frac{M}{J}
$$

where, $\ddot{\theta}$ is the angular acceleration; $M$ is the resultant couple moment on the blades; $J$ is the polar moment of inertia with the boundary condition for the integration (Neumann and Dirichlet, respectively) [6]:

$$
\begin{aligned}
& v\left(t_{0}\right)=\mathrm{C} 1 \cdot t=\text { velocity at time zero }=0 \\
& s\left(t_{0}\right)=\mathrm{C} 2=\text { position at time zero }=0
\end{aligned}
$$

The resultant couple moment on the blades " $M$ " is calculated as follows (Eq. (2)):

$$
M=d \cdot F
$$

where, $d$ is the barycenter of the force; and $F$ for 2D and 3D model, respectively (Eqs. (3) and (4)):

$$
\begin{gathered}
F=\int_{l} \sigma_{f} d l \\
F=\int_{A} \sigma_{f} d A
\end{gathered}
$$

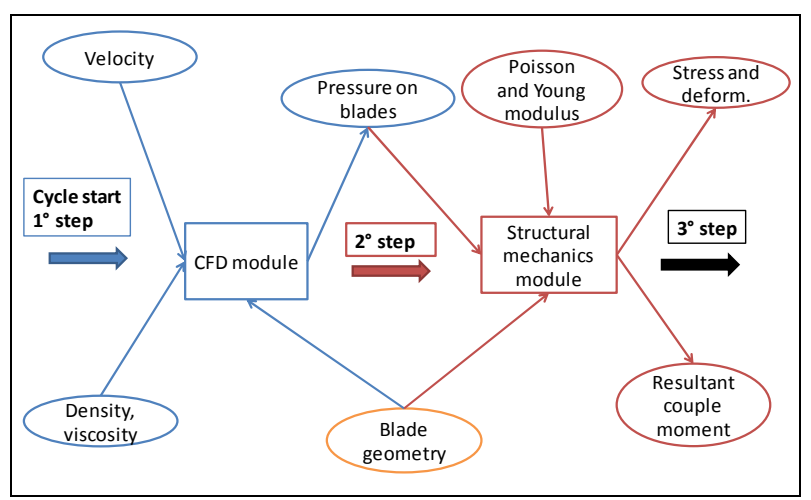

Fig. 3 Multiphysics model conceptual map (1/3). 
where, $\sigma_{f}$ is the stress caused by the FSI (fluid-structure interaction) and it is calculated by the Navier-Stokes equations; $d l$ and $d A$ are the frontal section and area of the blade in 2D and 3D model, respectively.

Also, the mesh rotation of the mobile domain follows the transformation matrix (matrix 1) [7]:

$$
\left[\begin{array}{l}
d x \\
d y
\end{array}\right]=\left[\begin{array}{cc}
\cos \vartheta & -\sin \vartheta \\
\sin \vartheta & \cos \vartheta
\end{array}\right] \cdot\left[\begin{array}{l}
x \\
y
\end{array}\right]
$$

where, $d x$ and $d y$ are the displacements of the mesh; $\vartheta$ is the mesh rotation angle, and it is calculated integrating the differential Eq. (1); $x$ and $y$ are the generic position of the mobile domain.

Finally, the moving mesh module will receive in input the turbine geometry, the rotation matrix and the rotation angle (that is the output of ODE module). As output, it will give the geometry in the new configuration generated by the turbine angular rotation (Fig. 4).

If the motion is fully developed the analysis stops, else the cycle re-starts at the $1^{\circ}$ step (Fig. 5).

Below, the multiphysics model functioning conceptual map is shown in Fig. 6.

This multiphysics model functioning conceptual map is implemented in COMSOL Multiphysics, both 2D and 3D.

Figs. 7 and 8 show the 2D model and 3D model in Comsol Multiphysics, respectively.

The choice between two or three-dimensional model follows this logic:

- If the blade geometry is representable on plane, (like Savonius turbine), the model will be 2D;

- If the blade geometry is not representable on plane, like axial turbine where the blade is warped, the model will be 3D.

Independently from fluid machinery, it is necessary to identify and to model the fix and the mobile mesh domain: the mobile domain is always constituted by blades and circular area that includes them (2D model in Fig. 7; 3D model, the circular area is replaced by cylinder or sphere in Fig. 8); the fix domain is constituted by rectangle/square (2D) or prism/cube (3D).

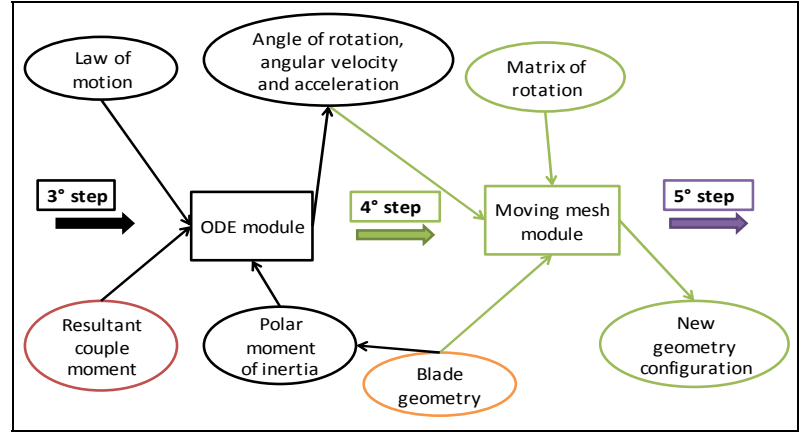

Fig. 4 Multiphysics model conceptual map (2/3).

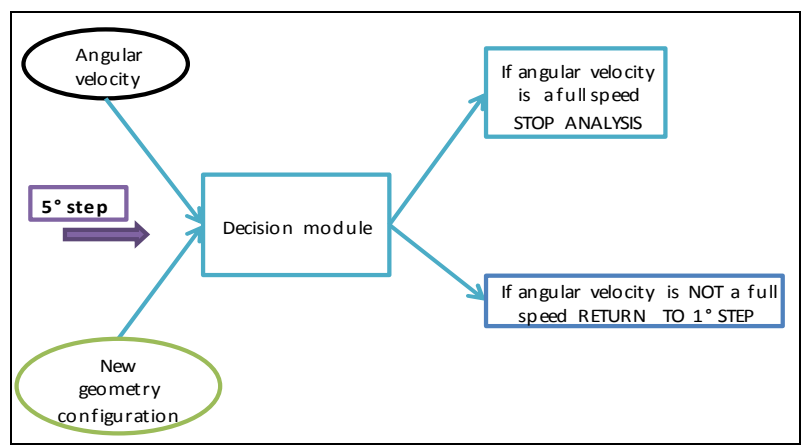

Fig. 5 Multiphysics model conceptual map (3/3).

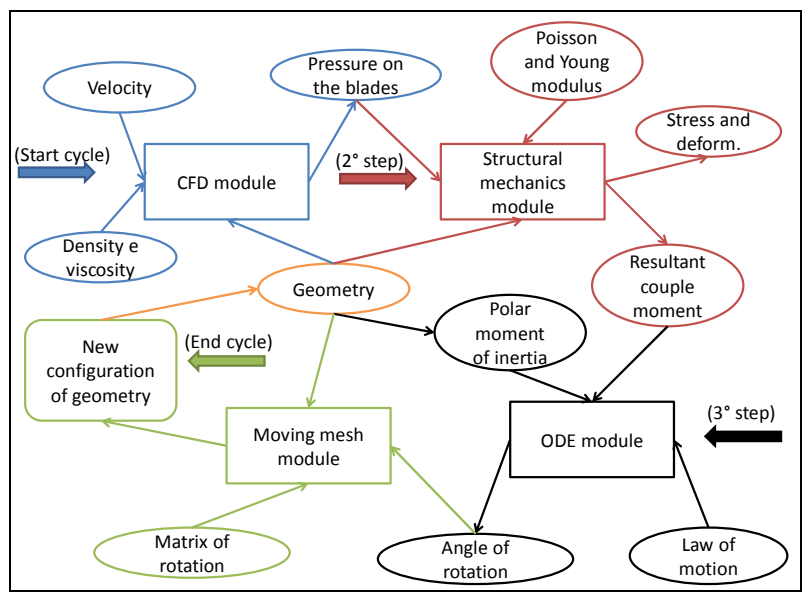

Fig. 6 Multiphysics model functioning conceptual map.

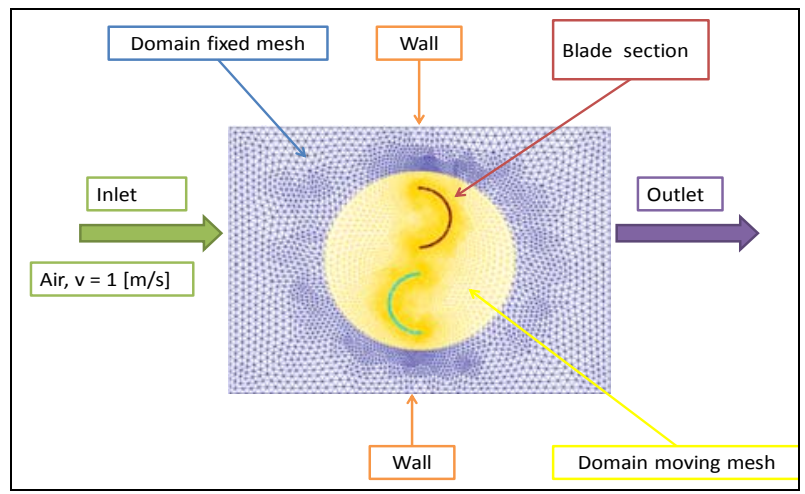

Fig. 7 2D model in Comsol Multiphysics. 


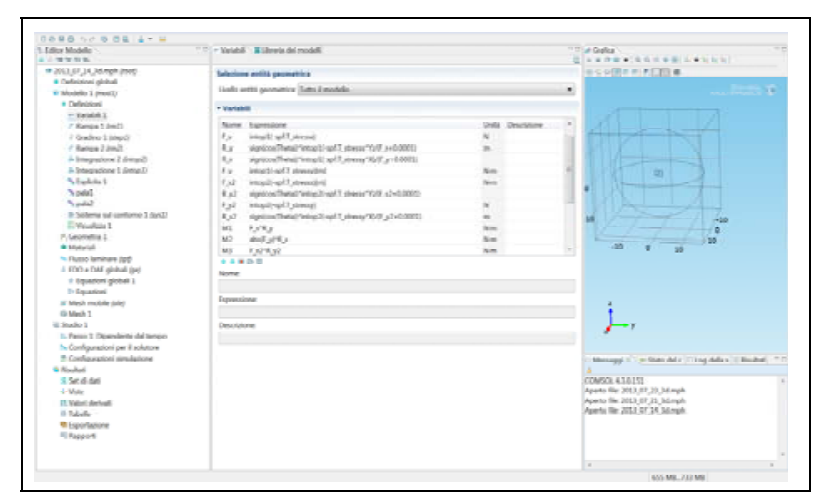

Fig. 8 3D model in Comsol Multiphysics.

\subsection{Mathematical Model Implementation}

After having completed the multiphysics model, it will be possible to extract the necessary data to realize the mathematical model. It has been developed through a mathematical software, easy to integrate with a CAD (MathCad Prime) and then it has been integrated with a CAD (Creo Parametric).

In the mathematical model (in MathCad Prime, as shown in Fig. 9), the validated results of the multiphysics numerical analysis are input; in this particular case, the $C_{d}$ value (the drag coefficient) has been extrapolated according to the various wind speeds and to the various blades dimensions and shapes [8]. Nota bene, the $C_{d}$ value (the drag coefficient) is evaluated both statically (with the turbine blades stopped) and dynamically (with the turbine blades moving).

In addition, there are included the equations required to calculate the minimum thickness with which to produce blades and the maximum producible theoretical power (Betz's limit-Eq. (5), maximum force-Eq. (6)) [9]:

$$
P_{\text {Betz }}=\frac{16}{27} F \cdot v
$$

where, $v$ is the fluid velocity; $F$ is calculated as follows (Eq. (6)):

$$
F=\frac{1}{2} \cdot \rho \cdot C_{d} \cdot A \cdot v^{2}
$$

where, $\rho$ is the fluid density; $C_{d}$ is the drag coefficient; $A$ is the frontal area; $v$ is the fluid velocity.

These equations are function of the material with

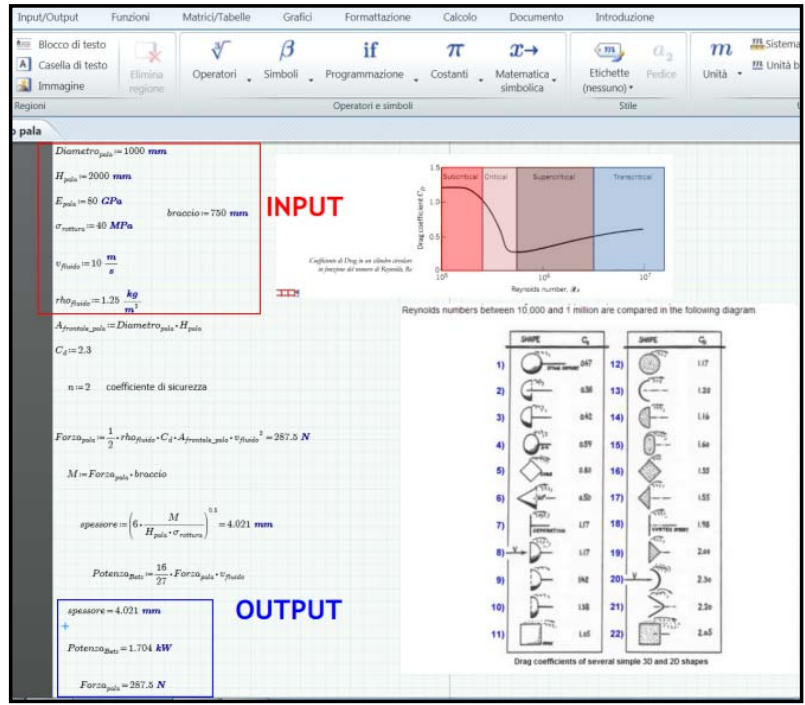

Fig. 9 Mathematic model in MathCad Prime.

which blades are manufactured, of the maximum wind speed to which they will be subjected, and of their dimensions (diameter and height) and shape.

\subsection{CAD Model}

In regard to the CAD model, once the geometry of turbine blade is designed, it will be necessary to link design parameters with CAD parameters which is input in the mathematical software (Fig. 10). There are two possibilities:

A. to link design parameters to CAD parameters;

B. to link CAD parameters to design parameters.

If we choose the first solution, modifications on the CAD model will occur modifying there the parameters directly (design parameters are updated, consequently); if we choose the second option, modifications on the

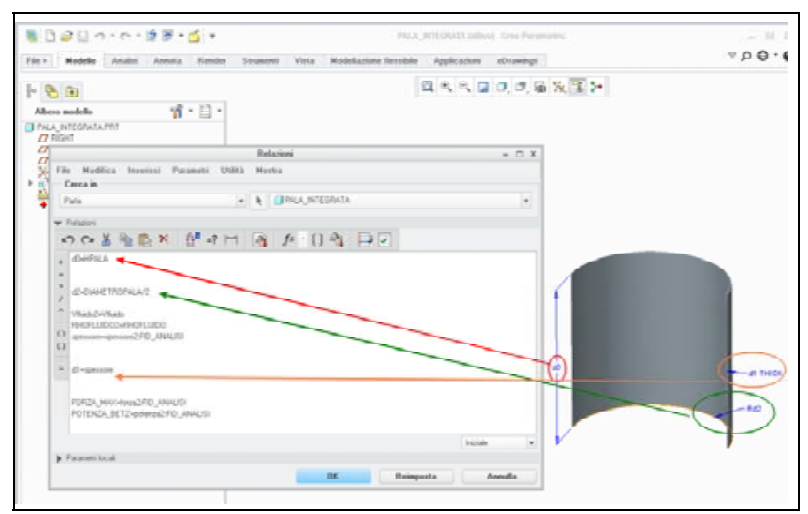

Fig. 10 Unidirectional link between Creo dimensions and parameters for MathCad. 
CAD model will be made indirectly through the modification of design parameters and CAD parameters will be automatically updated.

\section{Creo Parametric Integration with MathCad Prime}

After having realized both CAD model and mathematical model, it will be possible to integrate them. The most important operation is the parameters mapping, that is the creation of links between parameters created in CAD and parameters created in mathematical software. If a parameter has the same name, both in Creo and in MathCad (for example the parameter "blade diameter" has the same name in both software), Creo automatically creates the link, and mapping becomes automatic too (Figs. 11 and 12).

Differently, if parameters have different names in the two software (for example, if the parameter "blade diameter" has the name "Diameter" in Creo and "Diameter_Blade" in MathCad), the user will have to assign links between parameters in the two software. So, the user will have to select the desired parameter in Creo list (Fig. 13) and then he will select the corresponding parameter in MathCad list (Fig. 14).

\section{The Application of the Procedure}

The project manager modifies the blade diameter and its height, in modelling environment (e.g., in Creo Parametric), and selects the appropriate material and finally assigns the parameters related to maximum speed and density of the fluid. The MathCad Prime model returns the lowest value of thickness of the blade, the maximum theoretical extractable power and the maximum force to which blades are subjected (Fig. 15). In particular, in regard to the parameter of the lowest thickness of the blade, it is linked to the CAD parameter: 3D model will automatically update with the value of minimum thickness. At this point, the project manager has only to round up the thickness to the first useful "commercial" value. For example, if we would manufacture the blade with polycarbonate,

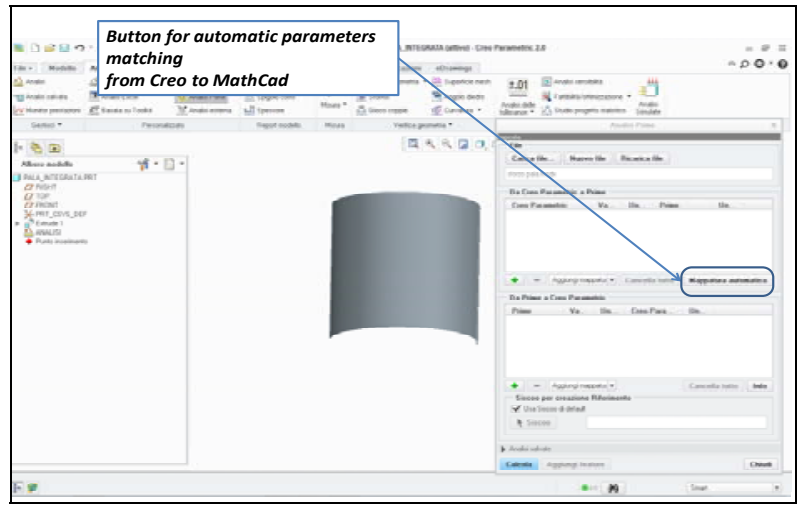

Fig. 11 Automatic parameters matching.

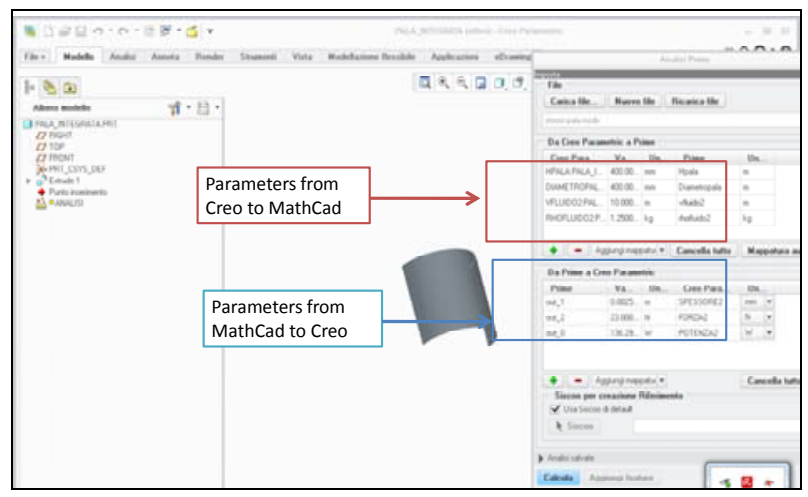

Fig. 12 Parameters matching Creo/MathCad.

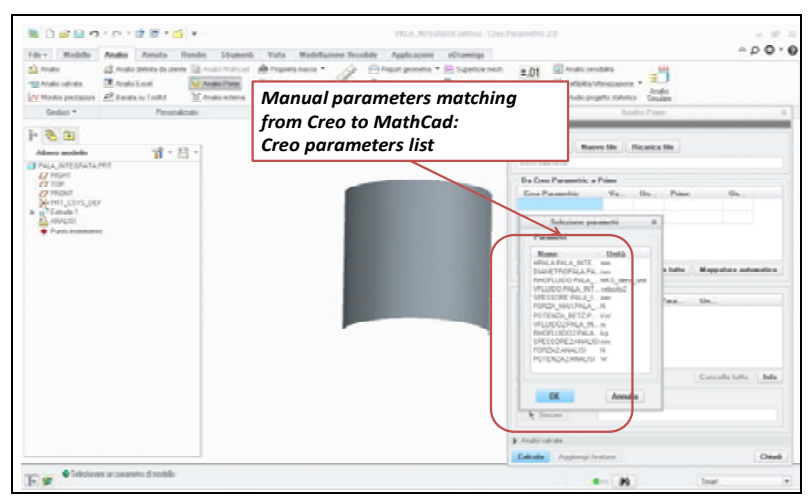

Fig. 13 Manual parameters matching-Creo parameters list.

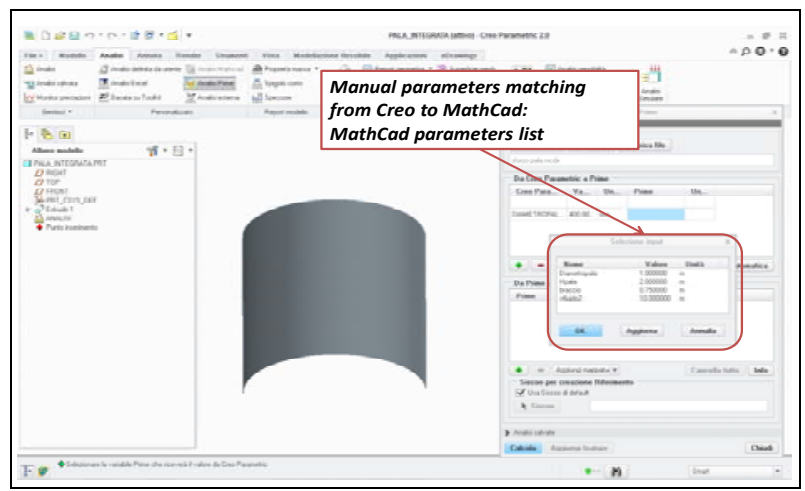

Fig. 14 Manual parameters matching-MathCad parameters list. 


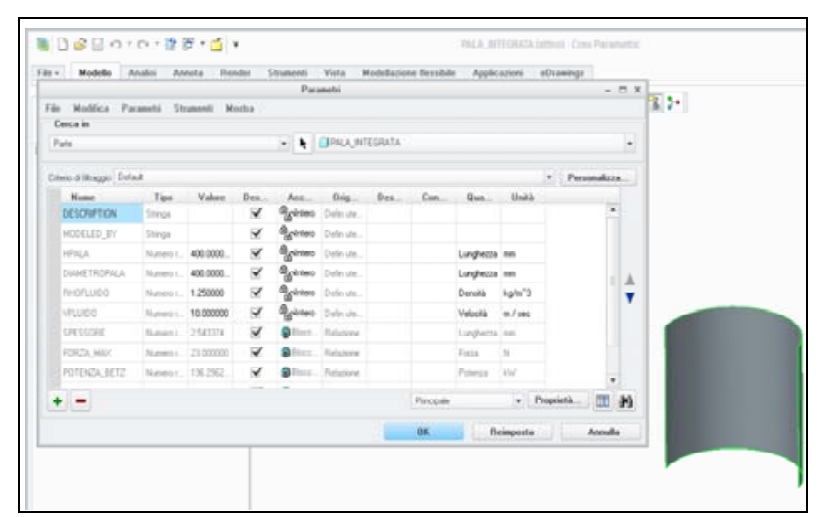

Fig. 15 Final parameters list in Creo Parametric.

and if the blade minimum thickness were $7.8 \mathrm{~mm}$, the project manager would have the task to round it up to $8 \mathrm{~mm}$, since commercial polycarbonate sheets of 8 $\mathrm{mm}$ have a greater diffusion and so a lower price than the $7.8 \mathrm{~mm}$ ones.

This procedure is easy and can be used even by the designer who does not know how to use a mathematical software (in particular MathCad Prime) and by who does not have knowledge about fluid dynamics, necessary for blade design.

\section{Conclusions and Future Work}

The proposed multiphysics model (realized in Comsol Multiphysics) has permitted to analyse a fluid machinery accurately, but there are the following complications: too much time for implementation, too much computational resource (for every simulation run) and too much CPU (central processing unit) time required (for every simulation run).

On the other hand, the mathematical model integrated with the CAD tool is suitable for preliminary sizing and it offers instant results (after its implementation); however, this method analyzes a fluid machinery less accurately and it does not permit to study the fluid machinery dynamics.

Therefore, if the aim is to design a new type of fluid machinery or optimize an existing fluid machinery, a multi physics model is necessary; on the contrary, if the aim is to choose the fluid machinery typology or its sizing, it is more suitable to use the mathematical model integrated with CAD.
With the aim to improve the just described design environment, the following improvements are in phase of study and implementation.

\subsection{CAD/CAE Integration}

The automatic integration between CAD and CAE models will be refined: once the integration of CAD model with CAE model will be completed, modifying CAD geometry, CAE geometry will be updated consequently, so the regeneration of various models from which the required data for the creation of mathematical model are acquired, would be faster.

\subsection{Integration with a Database of $C_{d}$, According to the Various Blade Shapes}

If we create a database with $C_{d}$ of the various typologies of commercial blade shapes, it will be possible to extend the use of the procedure not only to wind turbines, but also to the other typologies of fluid machines.

\subsection{Integration with a Database of Fluid Dynamic Properties of Various Fluids}

As a consequence of the extension to other typologies of fluid machines, it is necessary to create a database with fluid dynamic properties of different fluids; for example: air, water, vapour, etc.

\section{Acknowledgements}

The authors gratefully acknowledge Dr. Gionata Fragomeni of the University "Magna Graecia" of Catanzaro, for the use of Comsol Multiphysics and for sharing his insights in simulation-based design.

\section{References}

[1] Tornincasa, S., Bonisoli, E., and Di Monaco, F. 2011. "3D CAD and multi-domain simulation integration for Energy Scavenger design.” Presented at the International Conference on Innovative Methods in Product Design, Venice, Italy.

[2] Anderson, J. D. Jr. 2001. Fundamentals of Aerodynamics. 3rd edition. New York: McGraw-Hill.

[3] Braescu, L., and George, T. F. 2007. "Arbitrary Lagrangian-Eulerian Method for Coupled Navier-Stokes 
and Convection-Diffusion Equations with Moving Boundaries." In Proceedings of the 12th WSEAS International Conference on Applied Mathematics, 33-6.

[4] Bazilevs, Y., Hsu, M. C., Kiendl, J., Wuchner, R., and Bletzinger, K. U. 2011. "3D Simulation of Wind Turbine Rotors at Full Scale. Part II: Fluid-structure Interaction Modeling with Composite Blades.” International Journal for Numerical Methods in Fluids 65: 236-53.

[5] Stein, K., Tezduyar, T., and Benney, R. 2003 "Mesh Moving Techniques for Fluid-Structure Interactions with Large Displacements.” Journal of Applied Mechanics 70: 58-63.

[6] Funaro, D. 1992. "Polynomial Approximation of Differential Equations.” Lecture Notes in Physics. Vol. 8,
Berlin: Springer-Verlag, 182-90.

[7] Bazilevs, Y., Hsu, M. C., Akkerman, I., Wright, S., Takizawa, K., Henicke, B., Spielman, T., and Tezduyar, T. E. 2011. "3D Simulation of Wind Turbine Rotors at Full Scale. Part I: Geometry Modeling and Aerodynamics.” International Journal for Numerical Methods in Fluids 65: 207-35.

[8] McCormik, B. W. 1995. Aerodynamics, Aeronautics and Flight Mechanic. 2nd edition. New York: Wiley.

[9] Huleihil, M., and Mazor, G. 2012. "Wind Turbine Power: The Betz Limit and Beyond.” In Advances in Wind Power, edited by Carriveau, R. InTech. doi: $10.5772 / 52580$.

http://www.intechopen.com/books/advances-in-wind-po wer/wind-turbine-power-the-betz-limit-and-beyond. 